\title{
Loganic acid and anthocyanins from cornelian cherry (Cornus mas L.) fruits modulate diet-induced atherosclerosis and redox status in rabbits
}

\author{
Tomasz Sozański1,A-F, Alicja Z. Kucharska 2,B-F, Stanisław Dzimira 3,B,C,E,F, Jan Magdalann 1,B,C,E,F, Dorota Szumny 1,,,E,F, \\ Agnieszka Matuszewska ${ }^{1, C, E, F}$, Beata Nowak ${ }^{1, C, E, F}$, Narcyz Piórecki ${ }^{4,5, B, E, F}$, Adam Szeląg ${ }^{1, C, E, F}$, Małgorzata Trocha ${ }^{1, C, E, F}$ \\ ${ }^{1}$ Department of Pharmacology, Wroclaw Medical University, Poland \\ 2 Department of Fruit, Vegetable and Plant Nutraceutical Technology, Wrocław University of Environmental and Life Sciences, Poland \\ ${ }^{3}$ Department of Pathology, Wrocław University of Environmental and Life Sciences, Poland \\ ${ }^{4}$ Arboretum and Institute of Physiography in Bolestraszyce, Poland \\ ${ }^{5}$ Department of Tourism and Recreation, University of Rzeszów, Poland
}

A - research concept and design; B - collection and/or assembly of data; C - data analysis and interpretation;

$D$ - writing the article; $E$ - critical revision of the article; $F$ - final approval of the article

Address for correspondence

Tomasz Sozański

E-mail: tsoz@wp.pl

\section{Funding sources}

The study was financially supported by the stat-

utory means of Wroclaw Medical University

(ST.A080.17.035)

Conflict of interest

None declared

Received on March 6, 2017

Reviewed on May 31, 2017

Accepted on June 8, 2017

\begin{abstract}
Background. Cornelian cherry (Cornus mas L.) is a plant growing in southeast Europe, in the past used in folk medicine. There are many previous publications showing the preventive effects of (poly)phenolic compounds, especially anthocyanins, on cardiovascular diseases, but there is a lack of studies comparing the effects of (poly)phenolics and other constituents of fruits.

Objectives. We have attempted to determine if iridoids and anthocyanins from cornelian cherry fruits may affect the formation of atherosclerotic plaques in the aorta as well as lipid peroxidation and oxidative stress in the livers of cholesterol-fed rabbits.

Material and methods. Fractions of iridoids and anthocyanins were analyzed using the high-performance liquid chromatography (HPLC) and liquid chromatography-mass spectrometry (LC-MS) methods. Loganic acid $(20 \mathrm{mg} / \mathrm{kg} \mathrm{bw}$.) and a mixture of anthocyanins $(10 \mathrm{mg} / \mathrm{kg}$ bw.) were administered orally for 60 days to rabbits fed with $1 \%$ cholesterol. Histopathological samples of the aortas and the livers were stained with hematoxylin and eosin. Lipid peroxidation (malondialdehyde - MDA) and redox status (glutathione - GSH, glutathione peroxidase - Gpx and superoxide dismutase - SOD) were analyzed using spectrophotometrical methods.

Results. Both loganic acid (an iridoid) and a mixture of anthocyanins diminished the formation of atherosclerotic plaques in the aorta. Both substances also diminished lipid peroxidation, measured as a decrease of MDA, and attenuated oxidative stress, measured as an increase of GSH in the livers depleted by cholesterol feeding. Unexpectedly, cholesterol feeding decreased the Gpx activity in the liver, which was reversed by both investigated substances.
\end{abstract}

Conclusions. We have shown that both iridoids and anthocyanins help prevent fed-induced atherosclerosis, and the consumption of fruits rich in these substances may elicit beneficial effects on the cardiovascular system.

Key words: atherosclerosis, glutathione, anthocyanins, cornelian cherry, loganic acid

DOI

10.17219/acem/74638

Copyright

Copyright by Author(s)

This is an article distributed under the terms of the

Creative Commons Attribution Non-Commercial License

(http://creativecommons.org/licenses/by-nc-nd/4.0/) 


\section{Introduction}

Cardiovascular diseases are the leading causes of morbidity and mortality in both developing and developed countries. ${ }^{1}$ Oxidative stress and subsequent lipid peroxidation contribute to the development and progression of atherogenesis and other tissue damage. ${ }^{2,3}$ They also interact with other factors, like proinflammatory cytokines or substances the affecting nitric oxide (NO) synthesis, contributing to vascular pathologies from endothelial dysfunction to clinical symptoms of cardiovascular diseases. ${ }^{4}$

It is also widely accepted that diet, especially a diet consisting of large amounts of fruits and vegetables, may prevent the development and progression of cardiovascular diseases. ${ }^{5,6}$ There are many studies on both humans and animals showing the preventive effects of (poly)phenolic compounds - flavonoids, and especially their subclass - anthocyanins, on atherosclerosis and cardiovascular diseases. ${ }^{7-9}$ In contrast, we have not found any surveys comparing the effects and interactions of different constituents of plants, particularly fruits, on the risk factors and symptoms of cardiovascular diseases. Moreover, there are only a few studies comparing the potency of various flavonoids on high-fat diet-induced changes. ${ }^{10}$ In recent decades there has been increasing interest in iridoids, a wide group of plant constituents with possible pleiotropic effects on human metabolism. ${ }^{11}$ Although both anthocyanins or iridoids are common constituents of plants, only a few edible fruits contain pronounced amounts of iridoids or both substances. In the present study, we focused on cornelian cherry (Cornus mas L.), a plant growing or cultivated in Europe and southwest Asia, and cultivated in North America; widely consumed in some countries and, in the past, used in folk medicine. In our previous studies we proved that the European cultivar of cornelian cherry contains large amounts of iridoids mainly loganic acid - and anthocyanins. We also showed the preventive effects of whole fruits on diet-induced atherosclerosis in animals, through the activation of peroxisome proliferator-activated receptors alpha (PPAR $\alpha){ }^{12,13}$ In another study, using the same model of diet-induced atherosclerosis we showed that isolated loganic acid and anthocyanins decreased dyslipidemia, increased the expression of both PPAR $\alpha$ and peroxisome proliferatoractivated receptors gamma (PPAR $\gamma$ ) in the liver, and decreased the intima thickness and the intima/media ratio in the thoracic aortas. ${ }^{14}$
The aim of the present investigation was to compare the effects of loganic acid and anthocyanins isolated from cornelian cherry on the formation of atherosclerosis in the thoracic and abdominal aorta, measured by histopathological examination, as well as on lipid peroxidation and redox state in the liver, measured as the malondialdehyde (MDA), glutathione (GSH), glutathione peroxidase (Gpx), and superoxide dismutase (SOD) levels.

\section{Material and methods}

\section{Plant material and the preparation of samples of loganic acid and anthocyanins}

Cornelian cherry fruits were harvested in the Arboretum and Institute of Physiography in Bolestraszyce, Poland. The plant material was verified by Prof. Jakub Dolatowski, the voucher specimen (BDPA 3 967) is stored at the Herbarium of the Arboretum and Institute of Physiography in Bolestraszyce. Loganic acid and a mixture of anthocyanins were prepared in the Department of Fruit, Vegetable and Plant Nutraceutical Technology at Wrocław University of Environmental and Life Science (Poland), according to Kucharska et al.'s instructions with minor modifications. ${ }^{13}$

Juice from cornelian cherry fruits was purified using an Amberlite XAD-16 resin column (Rohm and Haas, Chauny Cedex, France). Iridoid and anthocyanin compounds present in the juice were eluted with ethanol/ water/acetic acid (80:19.9:0.1, v/v/v). Purified compounds were concentrated, using a Rotavapor rotary evaporator (Büchi, Flawil, Switzerland) in a water bath at $40^{\circ} \mathrm{C}$, and then dried in an SPT-200 vacuum dryer (ZUT Colector, Kraków, Poland) $\left(40^{\circ} \mathrm{C}, 00.094 \mathrm{MPa}\right)$. Dried compounds were fractionated by polyamide column chromatography $(150 \mathrm{~mm} \times 30 \mathrm{~mm})$ (Macherey-Nagel-CC 6.6, Düren, Germany), using ethanol/water/acetic acid (50:49.5:0.5, $\mathrm{v} / \mathrm{v} / \mathrm{v}$ ) as the eluent. As a result of separation, we obtained loganic acid and anthocyanins. The former was monitored at $254 \mathrm{~nm}$, while the latter - at $520 \mathrm{~nm}$ (Fig. 1). Next, the resulting fractions were concentrated with a Rotavapor rotary evaporator, and then dried in an SPT200 vacuum dryer $\left(40^{\circ} \mathrm{C}, 0.094 \mathrm{MPa}\right)$. The fractions were analyzed using the high-performance liquid chromatography (HPLC) (Fig.1) and liquid chromatography-mass spectrometry (LC-MS) methods.

Table 1. Schedule of animal feeding and administering test substances

\begin{tabular}{|l|l|}
\multicolumn{1}{|c|}{ Control group P } & \multicolumn{1}{c}{ Regular feed + saline p.o. } \\
\hline Experimental group $\mathrm{CHOL}$ & regular feed with the addition of $1 \%$ cholesterol + saline p.o. \\
\hline Experimental group $\mathrm{CHOL}+\mathrm{LA}$ & regular feed with the addition of $1 \%$ cholesterol + loganic acid $20 \mathrm{mg} / \mathrm{kg}$ bw. p.o \\
\hline Experimental group $\mathrm{CHOL}+\mathrm{ANT}$ & regular feed with the addition of $1 \%$ cholesterol + anthocyanins $10 \mathrm{mg} / \mathrm{kg}$ bw. p.o. \\
\hline
\end{tabular}




\section{The in vivo study}

This research was conducted in accordance with the National Institutes of Health (NIH) Guide for the Care and Use of Laboratory Animals and Directive 2010/63/EU, and was approved by the Local Ethical Committee on Animal Research at the Institute of Immunology and Experimental Therapy, Polish Academy of Sciences in Wrocław (Poland).

\section{Chemicals}

Loganic acid and anthocyanins were prepared as described above. Cholesterol was purchased from $\mathrm{POCH}$ (Gliwice, Poland).

\section{Animals and treatment}

Forty mature New Zealand rabbits aged 8-12 months were used in the study. The animals were housed in individual chambers with a temperature maintained at $21-$ $-23^{\circ} \mathrm{C}$ under a $12: 12 \mathrm{~h}$ light-dark cycle. During the experiment, the rabbits had free access to water and received the same daily portion of feed $(40 \mathrm{~g} / \mathrm{kg}$ bw.). After 4 weeks of acclimatization, the animals were divided by block randomization into 4 groups of 10 animals each. For 60 consecutive days of the experiment, the animals in group $\mathrm{P}$ ingested standard feed. The other groups - CHOL,

Table 2. Histopathological changes in the thoracic and abdominal aorta in rabbits in experimental groups - hematoxylin and eosin (HE) staining

\begin{tabular}{|c|c|c|c|c|}
\hline \multirow{2}{*}{$\begin{array}{c}\text { Thoracic } \\
\text { aorta }\end{array}$} & \multicolumn{4}{|c|}{ Group } \\
\hline & $\begin{array}{c}P \\
n=10\end{array}$ & $\begin{array}{l}\mathrm{CHOL}^{* *} \\
n=10\end{array}$ & $\begin{array}{c}{\mathrm{CHOL}+\mathrm{LA}^{\#}}^{n}=10\end{array}$ & $\begin{array}{c}\mathrm{CHOL}+\mathrm{ANT}^{\#} \\
n=10\end{array}$ \\
\hline- & 10 (100.0\%) & $1(10.0 \%)$ & 8 (80.0\%) & 9 (90.0\%) \\
\hline+ & $0(0.0 \%)$ & $5(50.0 \%)$ & $2(20.0 \%)$ & $1(10.0 \%)$ \\
\hline++ & $0(0.0 \%)$ & $2(20.0 \%)$ & $0(0.0 \%)$ & $0(0.0 \%)$ \\
\hline+++ & $0(0.0 \%)$ & $1(10.0 \%)$ & $0(0.0 \%)$ & $0(0.0 \%)$ \\
\hline++++ & $0(0.0 \%)$ & 1 (10.0\%) & $0(0.0 \%)$ & $0(0.0 \%)$ \\
\hline \multirow{2}{*}{$\begin{array}{c}\text { Abdominal } \\
\text { aorta }\end{array}$} & \multicolumn{4}{|c|}{ Group } \\
\hline & $\begin{array}{c}P \\
n=10\end{array}$ & $\begin{array}{l}\mathrm{CHOL}^{* * *} \\
n=10\end{array}$ & $\begin{array}{c}\mathrm{CHOL}+\mathrm{LA}^{*} \Delta \\
n=10\end{array}$ & $\begin{array}{c}\text { CHOL+ANT } \\
n=10\end{array}$ \\
\hline- & 10 (100.0\%) & $1(10.0 \%)$ & $5(50.0 \%)$ & 8 (80.0\%) \\
\hline+ & $0(0.0 \%)$ & $5(50.0 \%)$ & $5(50.0 \%)$ & $0(0.0 \%)$ \\
\hline++ & $0(0.0 \%)$ & $3(30.0 \%)$ & $0(0.0 \%)$ & $2(20.0 \%)$ \\
\hline++++ & $0(0.0 \%)$ & $1(10.0 \%)$ & $0(0.0 \%)$ & $0(0.0 \%)$ \\
\hline
\end{tabular}

Scoring: / - / no visible endothelium damage; / + / a few assembled foam cells occupying no more than $10 \%$ of the artery circumference; / ++/ 2 or more collections of atheromatous plaques; / +++ / atheromatous plaques occupying more than $2 / 3$ of the artery circumference and of thickness accounting for about $50 \%$ of the artery wall thickness; / ++++ / massive atheromatous plaques occupying all the artery circumference and of thickness exceeding $50 \%$ of the artery thickness. $\mathrm{P}$ - standard feed and vehicle-treated rabbits; $\mathrm{CHOL}$ - cholesterol-treated rabbits, $\mathrm{CHOL}+\mathrm{LA}$ - rabbits treated with cholesterol + loganic acid $20 \mathrm{mg} / \mathrm{kg}$ bw.; $\mathrm{CHOL}+\mathrm{ANT}$ - rabbits treated with cholesterol + anthocyanins $10 \mathrm{mg} / \mathrm{kg}$ bw. Values are presented as number and percentiles. Specific comparisons (the $X^{2}$ test): ${ }^{*} p<0.05$ vs $P$;

${ }^{* *} p<0.01$ vs $P ;{ }^{* * *} p<0.001$ vs $P ;{ }^{*} p<0.05$ vs CHOL; ${ }^{\Delta} p<0.05$ vs CHOL+ANT.
$\mathrm{CHOL}+\mathrm{LA}$ and $\mathrm{CHOL}+\mathrm{ANT}-$ received the same feed $+1 \%$ cholesterol. Once daily, in the morning, the following test substances were administered orally to the rabbits: groups $\mathrm{P}$ and $\mathrm{CHOL}$ - normal saline solution (negative and positive control), group $\mathrm{CHOL}+\mathrm{LA}-$ loganic acid at a dose of $20 \mathrm{mg} / \mathrm{kg}$ bw. and group CHOL+ANT - a mixture of anthocyanins at a dose of $10 \mathrm{mg} / \mathrm{kg}$ bw. (Table 1 ).

On the $0^{\text {th }}$ and $60^{\text {th }}$ days of study, blood samples were collected from each animal from the marginal vein of the ear, or the saphenous vein. On the $60^{\text {th }}$ day, the animals were euthanized with terminal anesthesia, using morbital (1 mL containing $133.3 \mathrm{mg}$ of sodium pentobarbital and $26.7 \mathrm{mg}$ of pentobarbital), administered intraperitoneally (ip.) at a dose of $2 \mathrm{~mL} / \mathrm{kg}$ bw. The organs were collected - the livers for the measurements of lipid peroxidation and oxido-redox state parameters, and the aortas for histopathological assessment.

\section{Biomarkers associated with oxido-redox state in the liver}

All parameters in the liver were assessed spectrophotometrically. The MDA level was assayed using the BIOXYTECH-MDA-586 kit (OxisResearch, Portland, USA) and expressed as $\mu \mathrm{M} / \mathrm{g}$ of tissue. The GSH concentration was assayed using the BIOXYTECH GSH-400 kit (OxisResearch) and expressed as $\mathrm{mM} / \mathrm{mg}$ of tissue. The SOD activity was measured using the Ransod kit (Randox Laboratories, Crumlin, UK) and expressed as U/mg of protein. The GPx activity was measured using the GPx-340 kit (Randox Laboratories) and expressed as $\mathrm{U} / \mathrm{mg}$ of protein.

\section{Histopathological evaluations}

Postmortem thoracic and abdominal aorta and liver samples were collected for histological examinations. Tissue sections were fixed in a $7 \%$ buffered formalin solution, embedded in paraffin, cut into $4 \mu \mathrm{m}$ thick slices, and stained by means of the hematoxylin and eosin method. The samples were blindly examined by an experienced pathomorphologist.

\section{Statistical analysis}

Histopathological results were expressed as the number (percentage) of animals presenting the same degree of changes. The $X^{2}$ test of independence was used to study the qualitative data. The results of all the other data were expressed as mean \pm standard deviation (mean \pm SD). The normality of all continuous variables was verified by the Shapiro-Wilk test. Statistical comparisons of histopathological data were performed using the Kruskall-Wallis test, followed by the Dunn test 


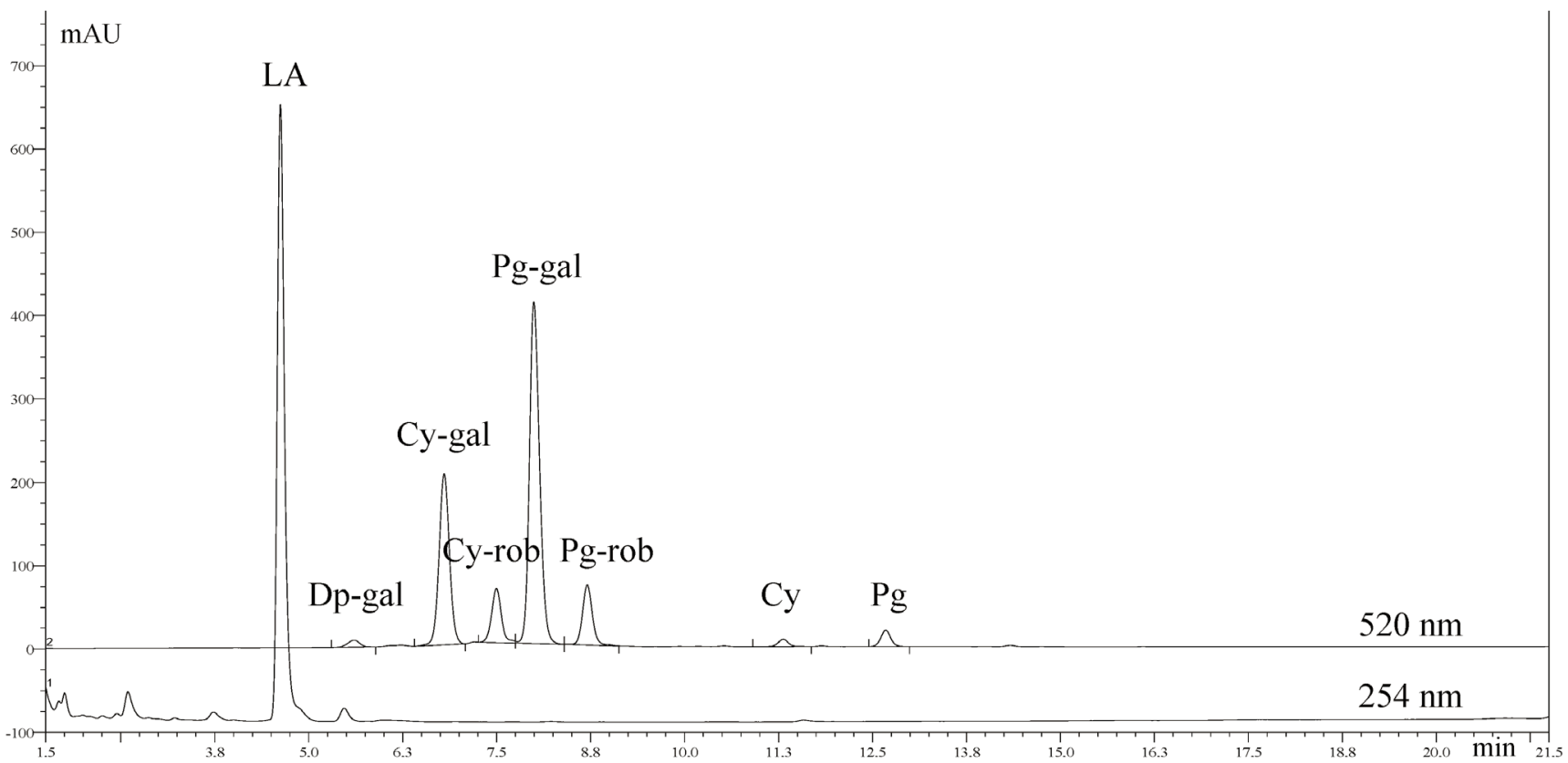

Fig. 1. High-performance liquid chromatography-diode-array detection (HPLC-DAD) chromatograms of iridoid (254 nm) and anthocyanins (520 nm) fractions from cornelian cherry (Cornus mas L.) fruits

LA - loganic acid; Dp-gal - delphinidin 3-O-galactoside; Cy-gal - cyanidin 3-O-galactoside; Cy-rob - cyanidin 3-O-robinobioside; Pg-gal - pelargonidin 3-O-galactoside; Pg-rob - pelargonidin 3-O-robinobioside; Cy - cyanidin; Pg - pelargonidin; mAU - milli absorbance units.

for multiple comparisons. Other data was analyzed using the analysis of variance (ANOVA), followed by a post-hoc least significant difference (LSD) test. The p-values $<0.05$ were considered statistically significant.

\section{Results}

We compared the impact of orally administered cornelian cherry constituents, an iridoid - loganic acid and anthocyanins, on dietary-induced hyperlipidemia in rabbits.

Both the thoracic and abdominal aortas in the control $\mathrm{P}$ group presented no atheromatous changes, whereas cholesterol-fed rabbits developed characteristic atheromatous lesions. There were plaques of different thickness, comprising lipid-loaded foam cells, lymphocytes, fibrin, and disintegrated cells with debris in the extracellular matrix. There were also visible mild proliferations of myocytes and the exudation in the subendothelial layer consisting of amorphous proteins and glycosaminoglycans. In a few cases, there were microclots on the tunica intima, composed of red blood cells and thrombocytes, occasionally incorporated into the arterial walls when surrounded by endothelium. Both loganic acid and anthocyanins prevented or significantly decreased the development of atheromatous changes (Table 2). In the thoracic aorta, in the $\mathrm{CHOL}+\mathrm{LA}$ and $\mathrm{CHOL}+\mathrm{ANT}$ groups, no lesions were noted in $80 \%$ and $90 \%$ of the rabbits, respectively. The remaining animals developed only mild changes with a few collections of assembled foam cells. The length of those changes did not exceed $10 \%$ of the circumference of the artery. In the abdominal aorta, in the CHOL+LA and $\mathrm{CHOL}+\mathrm{ANT}$ groups, no lesions were noted in 50\% and $80 \%$ of the rabbits, respectively. In the remaining $50 \%$ of the animals in the CHOL+LA group, only mild changes were observed, as described above. In only $20 \%$ of the rabbits in the $\mathrm{CHOL}+\mathrm{ANT}$ group, some atheromatous changes were noted, although they were moderate, with 2 or more plaques. Representative pictures showing histological cross-sections of the thoracic and abdominal aortas are presented in Fig. 2.

The cholesterol-enriched diet induced moderate fatty degeneration in the livers compared to the control group. Neither anthocyanins nor loganic acid significantly decreased those changes (data not presented).

Lipid peroxidation in the livers was measured by the MDA concentrations, and redox status by both enzymatic and non-enzymatic oxidative markers.

The MDA concentration in the CHOL group increased significantly in comparison to the control group. The addition of either anthocyanins or loganic acid significantly decreased lipid peroxidation. The MDA levels in the $\mathrm{CHOL}+\mathrm{ANT}$ and $\mathrm{CHOL}+\mathrm{LA}$ groups were comparable to each other and lower compared to the CHOL group, although they were still higher than in the group P. Simvastatin treatment completely reversed the increased MDA level caused by administering cholesterol (Fig. 3A).

Cholesterol-rich feeding significantly depleted the concentration of GSH. Both anthocyanins and loganic acid significantly increased the GSH level in comparison 

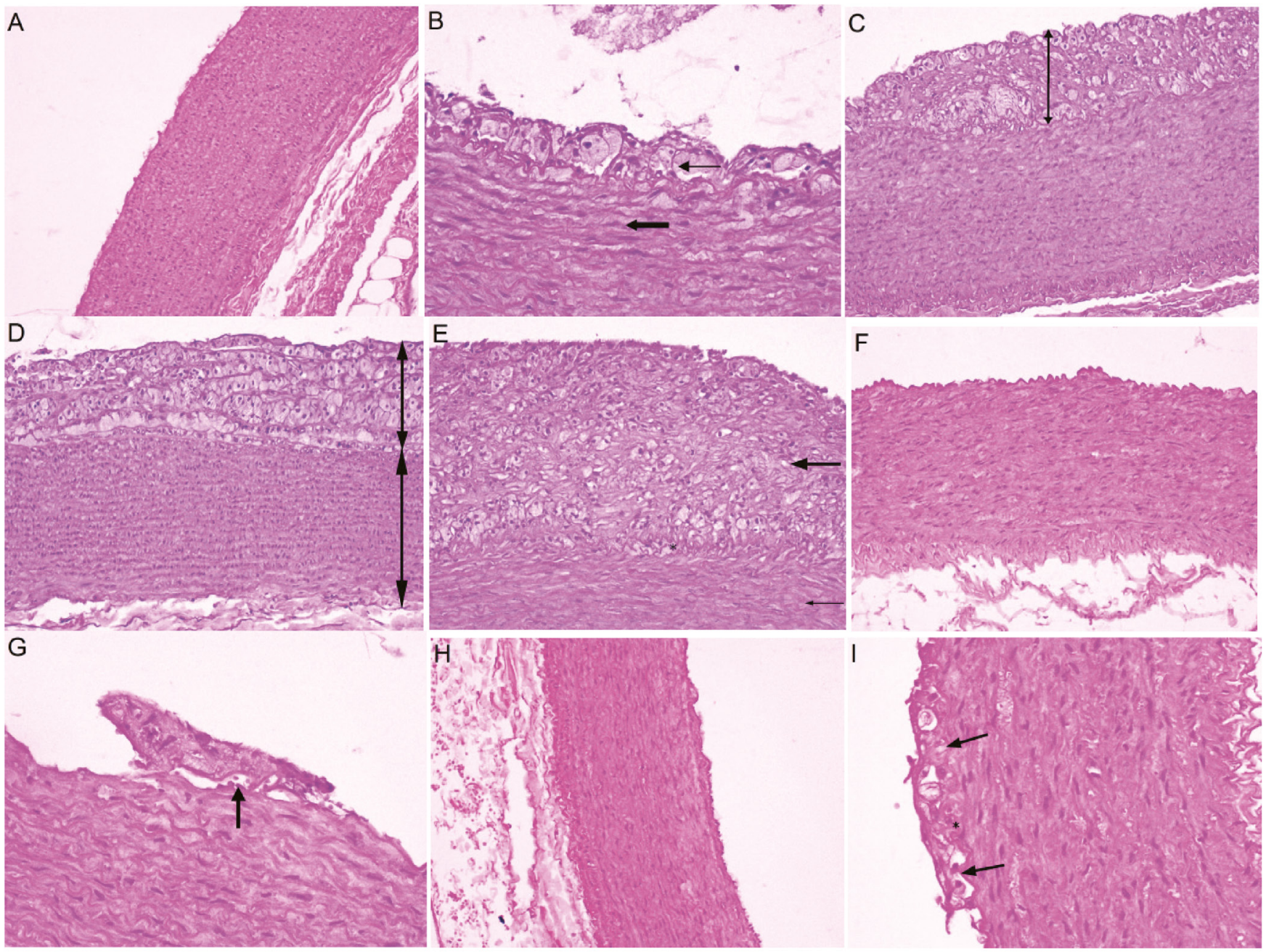

Fig. 2. Representative photos showing histological cross-sections of the aortas with hematoxylin and eosin (HE) staining

A - control group (rabbit No. 7). Normal endothelium and media of the thoracic aorta; changes classified as (-); HE staining, magnification $\times 100$. B - CHOL group (rabbit No. 18). A few assembled foam cells (thin arrow) on the endothelium of the abdominal aorta (thick arrow); changes classified as (+); HE staining, magnification $\times 200$. C - CHOL group (rabbit No. 19). A layer of atheromatous plaque (arrow) located on the endothelium of the thoracic aorta, composed of foam cells, a few lymphocytes and disintegrated cell debris; changes classified as (++); HE staining, magnification $\times 100$. D - CHOL group (rabbit No. 17). A thick layer of atheromatous plaque on the thoracic aorta endothelium, composed of numerous foam cells, a few lymphocytes and disintegrated cell debris; arrows indicate the thickness of the atheromatous plaque and the vessel; changes classified as (+++); HE staining, magnification $\times 100$. E - group CHOL (rabbit No. 14). A very thick layer of atheromatous plaque (thick arrow) on the abdominal aorta endothelium (thin arrow), composed of numerous foam cells, a few lymphocytes, myocytes, and disintegrated cell debris; changes classified as (++++); HE staining, magnification $\times 100$; * the border between the vessel and the plaque. F - CHOL+LA group (rabbit No. 21). Normal endothelium and media of the thoracic aorta; changes classified as (-); HE staining, magnification $\times 100$. G - CHOL+LA group (rabbit No. 26). Slightly detached endothelium (arrow) of the abdominal aorta; changes classified as (+); HE staining, magnification $\times 200$. H - CHOL+ANT group (rabbit No. 31). Normal endothelium and media of the thoracic aorta; changes classified as (-); HE staining, magnification $\times 100$. I - CHOL+ANT group (rabbit No. 39) Slightly detached endothelium (arrows) of the thoracic aorta; changes classified as (+); HE staining, magnification $\times 200$; * the border between the vessel and the plaque.

to the $\mathrm{CHOL}$ group; anthocyanins completely and significantly reversed the GSH depletion caused by cholesterol, although the loganic acid effect was weaker. The GSH level in the $\mathrm{CHOL}+\mathrm{LA}$ group was lower than in the $\mathrm{CHOL}$ group, but still higher than in the P group. Simvastatin completely prevented a decrease in the GSH concentration (Fig. 3B).

The GPx concentration in the CHOL group decreased compared to the control group, while the addition of either anthocyanins or loganic acid increased GPx activities to levels comparable to the control group (Fig. 3C).

SOD activities in the cholesterol-treated group increased significantly as compared to the control group.
The addition of neither anthocyanins nor loganic acid modulated these cholesterol-evoked changes. SOD activities in the $\mathrm{CHOL}+\mathrm{ANT}$ and $\mathrm{CHOL}+\mathrm{LA}$ groups were significantly higher compared to the control group, and comparable to the CHOL group (Fig. 3D).

\section{Discussion}

The most important finding of our study is that both iridoids and anthocyanins from the same plant may prevent atherosclerosis and redox stress. The key results are 

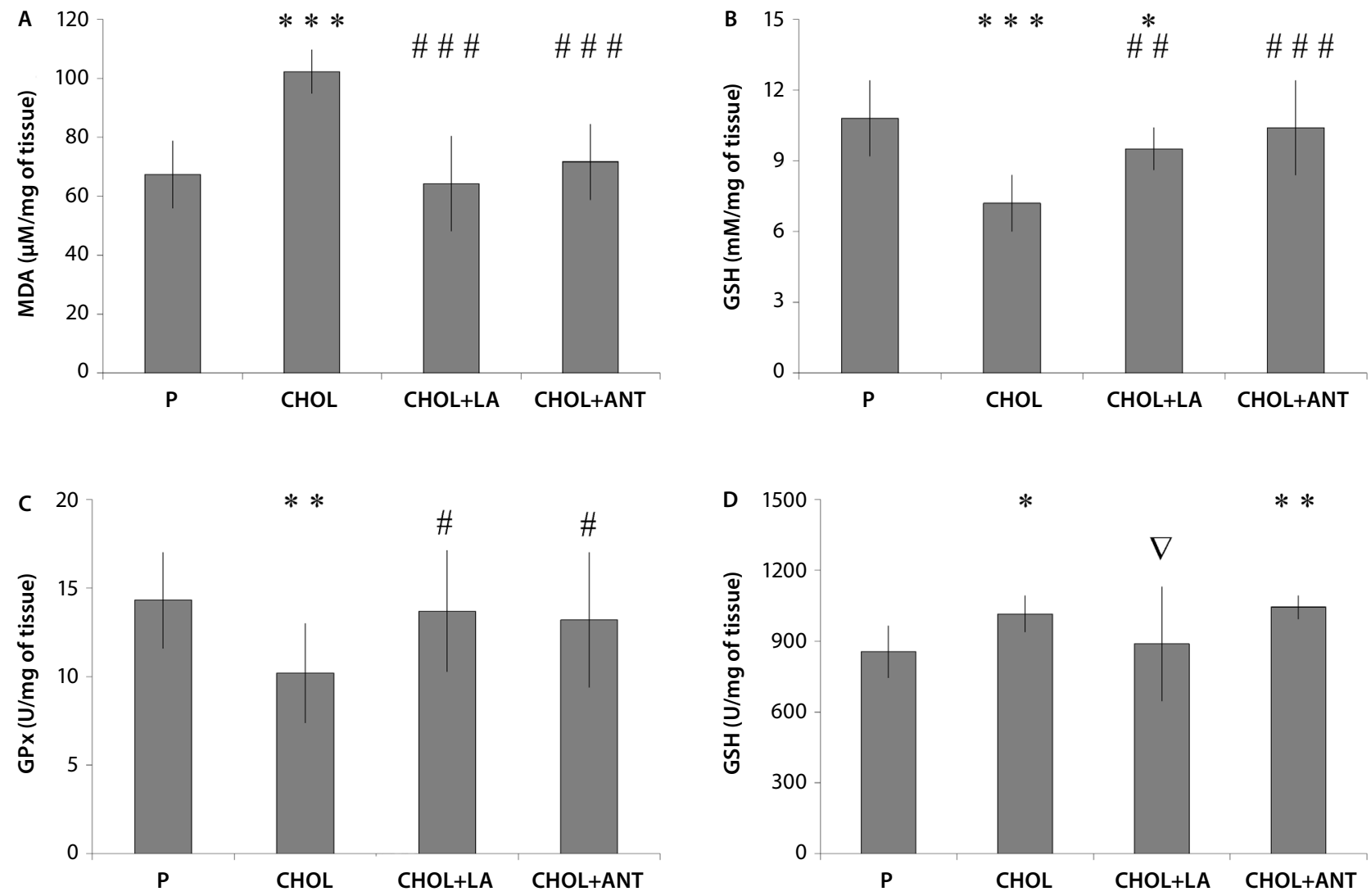

Fig. 3. The malondialdehyde (MDA) concentrations (A), glutathione (GSH) concentrations (B), glutathione peroxidase (GPx) activities (C), and superoxide dismutase (SOD) activities (D) in the liver

$\mathrm{P}$ - standard feed and vehicle-treated rabbits; CHOL - cholesterol-treated rabbits; CHOL+LA - rabbits treated with cholesterol + loganic acid $20 \mathrm{mg} / \mathrm{kg}$ bw:; CHOL+ANT - rabbits treated with cholesterol + anthocyanins $10 \mathrm{mg} / \mathrm{kg}$ bw.; values are presented as mean \pm SD. Specific comparisons: ${ }^{*} p<0.05$ vs $P$; ** $\mathrm{p}<0.01$ vs $\mathrm{P} ;{ }^{* * *} \mathrm{p}<0.001$ vs $\mathrm{P}$; $\# \mathrm{p}<0.05$ vs $\mathrm{CHOL}$; \#\# $\mathrm{p}<0.01$ vs $\mathrm{CHOL}$; \#\#\# $\mathrm{p}<0.001$ vs $\mathrm{CHOL} ; \triangle \mathrm{p}<0.05$ vs CHOL+ANT.

that both loganic acid, which belongs to a class of iridoids, and anthocyanins isolated from cornelian cherry fruits decreased the formation of atherosclerosis in the thoracic and, to a lesser extent, in the abdominal aorta of cholesterol-fed animals, while both substances decreased lipid peroxidation and oxidative stress in the liver.

Our finding that anthocyanins prevent atherosclerosis is consistent with numerous previous observations. Most of the available data attributes the cardiovascular benefits of fruits mainly to their (poly)phenolic fractions. A subclass of those, anthocyanins, abounds in cornelian cherry fruits. ${ }^{13,15}$ They are regarded as potent substances preventing atherosclerosis and cardiovascular diseases. ${ }^{8}$ Previous studies have found a higher content of anthocyanins in cornelian cherry fruits compared to other native or cultivated small fruits, such as raspberries, blackberries, red currants, and gooseberries, ${ }^{15,16}$ although the total content of anthocyanins may differ significantly among cultivars. ${ }^{17}$

Atherosclerosis is a complex pathological condition that engages various pathways, such as lipid oxidation and accumulation in the arteries, inflammation, redox stress, and NO depletion. Drugs and natural products used nowadays in the treatment of dyslipidemia, like 3-hydroxy-3-methyl glutaryl coenzyme A (HMG-CoA) reductase inhibitors (statins), fibric acid derivatives, bile acid-binding resins, and phytosterols, have quite different mechanisms of action. We hypothesize that fruits may also affect lipid metabolism and atherogenesis through their different constituents and through different mechanisms. Beyond the (poly)phenolic fraction, other constituents of plants generally regarded as contributing to the prevention of dyslipidemia and atherosclerosis are mono- and polyunsaturated fatty acids, soluble fiber, vegetable proteins, and phytosterols. ${ }^{18}$ Nevertheless, they are not found in substantial amounts in fruits.

As described in the Introduction, cornelian cherry fruits contain large amounts of anthocyanins, but are also abundant in iridoids. To the best of our knowledge, there is inadequate data comparing the biological effects and possible interactions between anthocyanins and iridoids, though those substances are present in the daily diet of western populations. In our previous studies, we detailed the constituents of cornelian cherry and proved that administering whole lyophilisated fruits diminished atherosclerosis through the same cholesterol-fed rabbit model we used in the present study. ${ }^{12,14,19}$ Subsequently, we found that both isolated loganic acid and anthocyanins contributed to the prevention of atherosclerosis, decreasing dislipidemia as well as decreasing the intima 
thickness and the intima/media ratio in the thoracic aorta of cholesterol-fed rabbits. We also revealed that both substances, especially anthocyanins, increased the expression of PPARs, while only iridoids possessed anti-inflammatory effects. ${ }^{13}$ At present, we completed a detailed evaluation of the formation of atherosclerosis in both the thoracic and abdominal aorta as well as the assessment of redox status in the livers, and proved that loganic acid contributes to the benefits we observed previously.

Iridoids that we investigated, including loganic acid, are a wide group of cyclopentan[c]pyran monoterpenoids, metabolites found in plants and animals. Previous studies have shown their potent biological effects, mainly their anti-inflammatory qualities. ${ }^{11}$ However, their preventive effects on diet-induced atherosclerosis, especially the possible mechanisms of action contributing to those effects, are not fully understood. This may be important, because some fruits, like those described in our studies - cornelian cherry, but also other members of the Cornus genus, especially the Asiatic Cornus officinalis L., and a few other fruits, contain large amounts of both anthocyanins and iridoids, although their qualitative and qualitative composition is different. ${ }^{14,20}$ The beneficial effects of iridoids on lipid metabolism and diabetes, with the modulation of PPAR $\alpha$ and sterol regulatory-element binding protein expression, were described regarding Cornus officinalis L., which grows mainly in Asia and has been used for years in traditional Chinese and Japanese medicine. ${ }^{21-23}$ However, Cornus officinalis L. contains different iridoids than the European cornelian cherry we investigated, mainly loganin and morroniside. The positive effects on lipids and atherosclerosis were also proven for other iridoids isolated from various plants. ${ }^{24-26}$ Nevertheless, knowledge about the effects of loganic acid we found in cornelian cherry on living systems, especially on atherogenesis, is poor.

In addition to the impact of loganic acid and anthocyanins on atherosclerosis, we also investigated their effects on lipid peroxidation and redox parameters in the liver. As we mentioned in the Introduction, there is a general conviction that oxidative stress plays a significant role in the pathogenesis of atherosclerotic vascular disease, proven by several in vitro and animal studies. ${ }^{2,3,27}$ Oxidative stress causes direct damage to molecules, lipid peroxidation, and affects inflammation (e.g., by the stimulation of inflammatory genes transcription), at the same time causing vascular dysfunction through the endothelial nitric oxide synthase (eNOS) depletion., ${ }^{4,28}$

We found that both loganic acid and anthocyanins exerted a preventive effect on lipid peroxidation through an impact on the MDA level, and that both substances restored depleted GSH levels in the liver. The effects of anthocyanins shown in the present study are consistent with previous in vitro and in vivo studies, in which both cornelian cherry and isolated anthocyanins showed antioxidant capacities. ${ }^{17,29,30}$ That observation seems to be supported by human studies that showed direct correlations between single anthocyanins intake and postprandial increases in serum antioxidant capacity. ${ }^{29}$ However, that increase was only temporary and probably cannot explain the impact of anthocyanins on atherosclerosis development.

There are also publications attributing antioxidant effects to iridoids. ${ }^{31}$ As we also described above, iridoids are a large group of substances that exert several effects on living systems, including substantial anti-inflammatory effects that may influence oxidative stress. ${ }^{11}$ Loganic acid and other iridoids also showed antiglycation activity, decreasing the production of advanced glycation end products (AGE), the elevation of which may be triggered by oxidative stress. ${ }^{32}$ However, we did not find any previous studies examining the direct effects of loganic acid on redox status.

Taking into account the doses of substances used in our experiment, human equivalent doses (HEDs) may be determined. According to the Food and Drug Administration, Center for Drug Evaluation and Research guidelines, ${ }^{33,34}$ HEDs of loganic acid and anthocyanins are $6.48 \mathrm{mg} / \mathrm{kg}$ bw. per day and $3.24 \mathrm{mg} / \mathrm{kg}$ bw. per day, respectively. For the human body weighing $100 \mathrm{~kg}$, HED of loganic acid is $648 \mathrm{mg} /$ day and of anthocyanins - $324 \mathrm{mg} /$ day. Estimated HEDs enable the creation of phytopharmaceuticals in solid dosage forms, such as capsules or tablets, as well as in liquid forms (both substances are soluble in water and in alcohol solutions). Moreover, taking into consideration the amount of loganic acid and anthocyanins in lyophilisated fruits, cornelian cherries may be used as a functional food. Daily HEDs of loganic acid and anthocyanins found in whole lyophilisated fruits (without a pit) are approx. $79 \mathrm{~g}$ and $137.4 \mathrm{~g}$, respectively (for a $100 \mathrm{~kg}$ man).

Based on our present and previous studies, possible indications for cornelian cherry and its isolated constituents include dyslipidemia and atherosclerosis. ${ }^{12,14,19}$ Whole dried or lyophilisated fruits may be used as a functional food in patients treated with a diet and lifestyle modifications alone. Isolated components or extracts may be used as phytopharmaceuticals in patients treated with statins. In our previous studies, we proved that both whole fruits and isolated constituents of cornelian cherry upregulated the expression of PPAR $\alpha$ in the liver. PPAR $\alpha$ is a transcriptional factor responsible for fatty acid catabolism via the activation of their beta-oxidation. ${ }^{12,14}$ We may speculate that due to a possibly different mechanism of pharmacological actions of cornelian cherry and its components and of statins, they may exert additive or synergistic effects.

Anthocyanins and iridoids are regarded as generally safe. Moreover, cornelian cherry fruits containing high amounts of those substances are considered edible and have been used in cuisine for hundreds of years. In our present and previous experiments, we have observed no adverse effects of 2-month-long oral administration of loganic acid, anthocyanins or whole lyophilisated fruits. Substances isolated from cornelian cherry used in our study, were not modified and, as stated above, their amounts may be consumed daily in dried or lyophilisated form by the general population. 
Here, the limitations of our study should be noted. Although we showed the preventive effects of the investigated substances on both atherosclerosis and oxidative stress, the assessment of a direct correlation is difficult, because there are many other factors influencing atherogenesis that we did not investigate. Moreover, although many in vitro and in vivo studies support the importance of redox modulation in tissues, their effects on clinical outcomes are unclear. Results of many human clinical trials involving the use of antioxidant vita mins are highly a mbiguous. ${ }^{35}$ Analyzing the changes in redox status, we should ask if the observed changes in lipid peroxidation and redox state are the direct results of the treatment and contribute to the positive effects on lipids and atherosclerosis. Alternatively, the antioxidant effects of the investigated substances may only be secondary changes related to other mechanisms - like the PPAR pathway or anti-inflammatory effects - subsequently diminishing redox triggering factors. This possibility remains a puzzle in several risk factors contributing to the development of atherosclerosis.

\section{Conclusions}

In conclusion, we have shown that different constituents - anthocyanins and iridoid loganic acid - of the same cornelian cherry plant showed the similar beneficial effects on holesterol-fed induced atherosclerosis. Anthocyanins and loganic acid diminished the formation of atherosclerotic plaques in the thoracic and, to a lesser extent, in the abdominal aorta, as well as diminished lipid peroxidation and reversed cholesterol-induced depletion of GSH in the liver. The results of our study indicate some potential benefits of consuming fruits containing both anthocyanins and iridoids that prevent cardiovascular diseases. Those results may also be applied in future attempts to produce phytopharmaceuticals containing those substances.

\section{References:}

1. Townsend N, Nichols M, Scarborough P, Rayner M. Cardiovascular disease in Europe: Epidemiological update. Eur Heart $\mathrm{J}$. 2015;36(40):2696-2705.

2. He F, Zuo L. Redox roles of reactive oxygen species in cardiovascular diseases. Int J Mol Sci. 2015;16(11):27770-27780.

3. Daiber A, Di Lisa F, Oelze M, et al. Crosstalk of mitochondria with NADPH oxidase via reactive oxygen and nitrogen species signaling and its role for vascular function [published online on December 9 , 2015]. Br J Pharmacol. 2015. doi:10.1111/bph.13403

4. Daiber A, Mader M, Stamm P, et al. Oxidative stress and vascular function. Cell Membranes and Free Radical Research. 2013;5(1):221-232.

5. Henkin Y, Kovsan J, Gepner Y, Shai I. Diets and morbid tissues: History counts, present counts. Br J Nutr. 2015;113(Suppl 2):S11-18.

6. Stefler D, Malyutina S, Kubinova R, et al. Mediterranean diet score and total and cardiovascular mortality in Eastern Europe: the HAPIEE study. Eur J Nutr. 2017;56(1):421-429.

7. Tsuda T. Dietary anthocyanin-rich plants: Biochemical basis and recent progress in health benefits studies. MolNutr Food Res. 2012;56(1):159-170.

8. Cassidy A, Mukamal KJ, Liu L, Franz M, Eliassen AH, Rimm EB. High anthocyanin intake is associated with a reduced risk of myocardial infarction in young and middle-aged women. Circulation. 2013;127(2):188-196.
9. Rangel-Huerta OD, Pastor-Villaescusa B, Aguilera CM, Gil A. Systematic review of the efficacy of bioactive compounds in cardiovascular disease: Phenolic compounds. Nutrients. 2015;7(7):5177-5216.

10. Hoek-van den Hil EF, van Schothorst EM, van der Stelt I, et al. Direct comparison of metabolic health effects of the flavonoids quercetin, hesperetin, epicatechin, apigenin and anthocyanins in high-fat-dietfed mice. Genes and Nutrition. 2015;10(4):469.

11. Viljoen A, Mncwangi N, Vermaak I. Anti-inflammatory iridoids of botanical origin. Curr Med Chem. 2012;19(14):2104-2127.

12. Sozański T, Kucharska AZ, Szumny A, et al. The protective effect of the Cornus mas fruits (cornelian cherry) on hypertriglyceridemia and atherosclerosis through PPARa activation in hypercholesterolemic rabbits. Phytomedicine. 2014;21(13):1774-1784.

13. Kucharska AZ, Szumny A, Sokół-Łętowska A, Piórecki N, Klymenko SV. Iridoids and anthocyanins in cornelian cherry (Cornus mas L.) cultivars. J Food Comp Anal. 2015;40:95-102.

14. Sozański T, Kucharska AZ, Rapak A, et al. Iridoid-loganic acid versus anthocyanins from the Cornus mas fruits (cornelian cherry): Common and different effects on diet-induced atherosclerosis, PPARs expression and inflammation. Atherosclerosis. 2016;254:151-160.

15. Pantelidis GE, Vasilakakis M, Manganaris GA, Diamantidis G. Antioxidant capacity, phenol, anthocyanin and ascorbic acid contents in raspberries, blackberries, red currants, gooseberries and cornelian cherries. Food Chem. 2007;102:777-783.

16. Vareed SK, Reddy MK, Schutzki RE, Nair MG. Anthocyanins in Cornus alternifolia, Cornus controversa, Cornus kousa and Cornus florida fruits with health benefits. Life Sci. 2006;78(7):777-784.

17. Popović BM, Stajner D, Slavko K, Sandra B. Antioxidant capacity of cornelian cherry (Cornus mas L.): Comparison between permanganate reducing antioxidant capacity and other antioxidant methods. Food Chem. 2012;134(2):734-741.

18. Rosa COB, dos Santos CA, Leite Jl, Caldas AP, Bressan J. Impact of nutrients and food components on dyslipidemias: What is the evidence? Adv Nutr. 2015;6(6):703-711.

19. Sozański T, Kucharska AZ, Szumny D, et al. Cornelian cherry consumption increases the L-arginine/ADMA ratio, lowers ADMA and SDMA levels in the plasma, and enhances the aorta glutathione level in rabbits fed a high-cholesterol diet. J Funct Foods. 2017;34:189-196.

20. Du WF, Cai H, Wang MY, Ding X, Yang H, Cai BC. Simultaneous determination of six active components in crude and processed Fructus Corni by high performance liquid chromatography. J Pharm Biomed Anal. 2008;48(1):194-197.

21. Park CH, Yamabe N, Noh JS, Kang KS, Tanaka T, Yokozawa T. The beneficial effects of morroniside on the inflammatory response and lipid metabolism in the liver of $\mathrm{db} / \mathrm{db}$ mice. Biol Pharm Bull. 2009;32(10):1734-740.

22. Park CH, Noh JS, Tanaka T, Yokozawa T. Effects of morroniside isolated from Corni Fructus on renal lipids and inflammation in type 2 diabetic mice. J Pharm Pharmacol. 2010;62(3):374-380.

23. Yamabe N, Noh JS, Park CH, et al. Evaluation of loganin, iridoid glycoside from Corni Fructus, on hepatic and renal glucolipotoxicity and inflammation in type 2 diabetic $\mathrm{db} / \mathrm{db}$ mice. Eur J Pharmacol. 2010;648(1-3):179-187.

24. Liao P, Liu L, Wang B, Li W, Fang X, Guan S. Baicalin and geniposide attenuate atherosclerosis involving lipids regulation and immunoregulation in ApoE-/- mice. Eur J Pharmacol. 2014;740:488-495.

25. Liu JY, Zhang DJ. Amelioration by catalpol of atherosclerotic lesions in hypercholesterolemic rabbits. Planta Med. 2015;81(3):175-184.

26. Gao Y, Chen ZY, Liang X, Wei SF. Anti-atherosclerotic effect of geniposidic acid in a rabbit model and related cellular mechanisms. Pharm Biol. 2015;53(2):280-285.

27. Kang DH, Kang SW. Targeting cellular antioxidant enzymes for treating atherosclerotic vascular disease. Biomol Ther (Seoul). 2013;21(2):89-96. doi:10.4062/biomolther. 2013.015

28. Aboonabi A, Singh I. Chemopreventive role of anthocyanins in atherosclerosis via activation of Nrf2-ARE as an indicator and modulator of redox. Biomed Pharmacother. 2015;72:30-36.

29. Mazza G, Kay CD, Cottrell T, Holub BJ. Absorption of anthocyanins from blueberries and serum antioxidant status in human subjects. J Agric Food Chem. 2002;50(26):7731-7737.

30. Rafieian-Kopaei M, Asgary A, Adelnia M, et al. The effects of cornelian cherry on atherosclerosis and atherogenic factors in hypercholesterolemic rabbits. J Med Plants Res. 2011;5:2670-2676. 
31. Barreto RS, Albuquerque-Júnior RL, Araújo $A A$, et al. A systematic review of the wound-healing effects of monoterpenes and iridoid derivatives. Molecules. 2014;19(1):846-862.

32. West BJ, Uwaya A, Isami F, Deng S, Nakajima S, Jensen CJ. Antiglycation activity of iridoids and their food sources. Int J Food Sci. 2014;2014:276950. doi:10.1155/2014/276950

33. U.S. Department of Health and Human Services, Food and Drug Administration, Center for Drug Evaluation and Research (CDER). Guidance for Industry. Estimating the Maximum Safe Starting Dose in Initial Clinical Trials for Therapeutics in Adult Healthy Volunteers. Chapter V. Step 2: Human equivalent dose calculation. Published by the Annual Review of Pharmacology and Toxicology, July 2005. https://www.fda.gov/downloads/drugs/guidances/ucm078932.pdf. Accessed April 16, 2016

34. Nair $A B$, Jacob $S$. A simple practice guide for dose conversion between animals and human. J Basic Clin Pharm. 2016;7(2):27-31.

35. Vogiatzi G, Tousoulis D, Stefanadis $C$. The role of oxidative stress in atherosclerosis. Hellenic J Cardiol. 2009;50(5):402-409. 